\title{
A RANDOMISED CROSSOVER STUDY OF LOW-FLOW AIR OR OXYGEN VIA NASAL \\ CANNULAE TO PREVENT DESATURATION IN PRETERM INFANTS
}

Conor C. Hensey MRCPI, ${ }^{1}$ Eoghan Hayden BSc, ${ }^{1}$ Colm P.F. O’Donnell PhD. ${ }^{1-3}$

1. The National Maternity Hospital, Holles Street;

2. National Children's Research Centre;

3. School of Medicine and Medical Science, University College Dublin; all Dublin, Ireland.

\section{Corresponding Author Dr. Colm O'Donnell}

Address

Dept. of Neonatology, The National Maternity Hospital, Holles

Street, Dublin 2, Ireland.

Email_codonnell@nmh.ie

Telephone +35316373100

Fax +35316614623

Sources of funding None Conflicts of interest None

Author Conor Hensey wrote the first draft of the manuscript and no honorarium, grant, or other form of payment was given to anyone to produce the manuscript.

Keywords infant; premature; respiration; oxygen; randomised

Word Count Abstract Main Text 
What is known about this topic:

Gas is commonly given via nasal cannulae to preterm infants at flow rates $<1 \mathrm{~L} / \mathrm{min}$ to prevent desaturation.

Infants may be given $100 \%$ oxygen; or lower oxygen concentrations may be given using an air/oxygen blender.

Opinions differ as to the relative contribution of the gas flow itself and the supplemental oxygen in reducing the frequency and/or severity of desaturation.

\section{What this study adds:}

At a flow rate of $0.1 \mathrm{~L} / \mathrm{min}$, air is no better than sham treatment at preventing desaturation, while $100 \%$ oxygen is superior to both .

This suggests that flow of gas is not important in the treatment of desaturation at this flow rate.

\section{ABSTRACT \\ Objective}


To compare the efficacy of low-flow oxygen, low-flow air, and sham treatment given via nasal cannulae in preventing desaturation (falls in oxygen saturation $\left[\mathrm{SpO}_{2}\right]$ ) in preterm infants.

\section{Study Design}

Infants born at $<33$ weeks gestation receiving gas at flow rates $<1 \mathrm{~L} / \mathrm{min}$ via nasal cannulae were eligible for inclusion. Enrolled infants received 3 treatments $-0.1 \mathrm{~L} / \mathrm{min} 100 \%$ oxygen, $0.1 \mathrm{~L} / \mathrm{min}$ air, ( $21 \%$ oxygen) and sham (tubing disconnected from flow meter) - via nasal cannulae, each for 3 hours. Treatments were given in a randomly assigned order and caregivers were masked to treatment. Infants were monitored with a pulse oximeter that recorded $\mathrm{SpO}_{2}$ and heart rate every 2 seconds. A treatment was stopped before 3 hours if infants reached pre-specified failure criteria. We compared the rates of failure and the frequency and duration of desaturation episodes that occurred during each treatment.

\section{Results}

Of 14 infants enrolled, 2 (14\%) reached failure criteria during treatment with oxygen compared to 7 (50\%) during treatment with both air and sham. Among infants who completed the 3-hour study periods, there were fewer episodes and shorter duration of desaturation with oxygen compared to both air and sham. 


\section{Conclusions}

At a flow rate of $0.1 \mathrm{~L} / \mathrm{min}$ via nasal cannulae, air is no better than sham treatment in preventing desaturation in preterm infants, while $100 \%$ oxygen is superior to both. 


\section{INTRODUCTION}

Gas is commonly given to spontaneously breathing preterm infants at low rates of flow $(<1 \mathrm{~L} / \mathrm{min})$ via nasal cannulae in an attempt to prevent falls in their oxygen saturation $\left(\mathrm{SpO}_{2}\right)$, or desaturation.[1] The use of nasal cannulae to deliver oxygen $\left(\mathrm{O}_{2}\right)$ was first proposed over 20 years ago,[2] yet it remains poorly studied. Infants may be given $100 \% \mathrm{O}_{2}$; or, alternatively, lower $\mathrm{O}_{2}$ concentrations may be given at low flow rates by using an air/ $\mathrm{O}_{2}$ blender in the circuit. A number of studies have calculated the effective fraction of inspired $\mathrm{O}_{2}\left(\mathrm{FiO}_{2}\right)$ for infants on low-flow blended air and $\mathrm{O}_{2}$ based on the infant's minute ventilation, the gas flow rate, and the percentage of $\mathrm{O}_{2}$ in the blended gas.[3,4] In a study of 187 preterm infants on low-flow nasal cannulae gas, the success of weaning to air during a challenge was associated with the effective $\mathrm{FiO}_{2}$ prior to the challenge.[5] In this study $27.8 \%$ of infants were receiving a calculated effective $\mathrm{FiO}_{2}$ of less than $0.23 .[5]$

Opinions differ as to the relative contribution of the gas flow itself and the supplemental $\mathrm{O}_{2}$ in reducing the frequency and/or severity of falls in $\mathrm{SpO}_{2}$. We wished to compare the effect of low-flow air to that of $100 \% \mathrm{O}_{2}$ in preventing episodes of desaturation. Thus, we determined whether the use of low-flow $100 \% \mathrm{O}_{2}$ compared to low-flow air $\left(21 \% \mathrm{O}_{2}\right)$, and to sham treatment, resulted in fewer falls in $\mathrm{SpO}_{2}$ in caffeine-treated infants born at $<33$ weeks gestation who were receiving supplemental oxygen through nasal cannulae for $\mathrm{SpO}_{2}$ $<90 \%$ in air. 


\section{METHODS}

We performed this randomised crossover study at The National Maternity Hospital, Dublin with the approval of our Research Ethics Committee between September 2009 and June 2010. The National Maternity Hospital is a stand-alone university maternity hospital with approximately 10,000 deliveries per year and a tertiary level neonatal unit. Infants at our hospital receive supplemental $\mathrm{O}_{2}$ targeting $\mathrm{SpO}_{2}$ in the range $88-95 \%$ and wean directly from nasal continuous positive airways pressure (NCPAP) to room air, with low-flow supplemental $\mathrm{O}_{2}$ via nasal cannulae reserved for those who do not maintain $\mathrm{SpO}_{2}$ in the desired range. Before this study, either $100 \% \mathrm{O}_{2}$ was given at a low-flow rate or an air $/ \mathrm{O}_{2}$ mixture determined with a blender was given at a relatively higher flow rate at the discretion of the treating clinician. At our hospital, gas given a $<1 \mathrm{~L} / \mathrm{min}$ is neither heated nor humidified, high-flow (i.e. $>1 \mathrm{~L} / \mathrm{min}$ ) nasal cannulae $\mathrm{O}_{2}$ is not routinely used and premature infants receive caffeine until 34 weeks corrected gestational age. Infants were eligible for the study if they were born at $<33$ weeks gestation, were considered clinically stable off NCPAP for $>48$ hours by the treating clinicians, and were receiving gas by nasal cannulae at a flow rate of $<1 \mathrm{~L} / \mathrm{min}$. Infants were excluded if they had a major congenital anomaly, or had ongoing apnea, bradycardia, haemodynamic instability, or other illnesses that could cause desaturation. Written consent was obtained from parents by a member of the research team to enrol their infants into the study.

All infants received gas via nasal cannulae (Premature curved prong with $2.1 \mathrm{~m}$ tubing, Intersurgical, Wokingham, England) at a flow rate determined using a flow meter (Timeter, 
St Louis MO, USA) and an $\mathrm{O}_{2}$ concentration determined with an air $/ \mathrm{O}_{2}$ blender (VIP Bird, Bird Products Corporation, Palm Springs CA, USA). Each infant received each of the 3 treatments below for consecutive 3 hour periods (i.e. total study time of 9 hours):

- $\quad$ Flow meter set to $0.1 \mathrm{~L} / \mathrm{min}$, blender set to $100 \% \mathrm{O}_{2}$ (oxygen, $\mathrm{O}$ )

- $\quad$ Flow meter set to $0.1 \mathrm{~L} / \mathrm{min}$, blender set to $21 \% \mathrm{O}_{2}$ (air, $\mathrm{A}$ )

- $\quad$ Flow meter turned off, blender set to $21 \% \mathrm{O}_{2}$, nasal cannulae disconnected from flow meter (sham, S)

We chose the flow rate of $0.1 \mathrm{~L} / \mathrm{min}$ as it is commonly used in our unit. Infants were randomly assigned to receive these treatments in the order $\mathrm{O}-\mathrm{S}-\mathrm{A}$ or $\mathrm{A}-\mathrm{S}-\mathrm{O}$ using a random number table. One member of the research team made all adjustments to the flow meter and blender and securely fastened an opaque cover over them in order to mask the treatment the infant was receiving from caregivers (Figure 1). All other aspects of the infant's treatment were unaffected.

All infants were monitored with a pulse oximeter (Masimo Radical 7, Masimo Corporation, Irvine CA, USA) with the sensor (LNOP Neo-L) placed on the hand or foot. The site of the sensor was not changed for the 9 hours of the study. The oximeter was set to record data $\left(\mathrm{SpO}_{2}\right.$ and heart rate, $\left.\mathrm{HR}\right)$ every 2 seconds with maximal sensitivity. The following alarm limits were set on the pulse oximeter for each infant and were not changed during the study: $\mathrm{SpO}_{2}$ - no upper alarm limit, lower alarm limit 85\%; HR - upper alarm limit 180bpm, 
lower alarm limit 100bpm. Enrolled infants were monitored with this pulse oximeter only for the duration of the study.

Our primary outcome was treatment failure. Enrolled infants were deemed to have failed a treatment if they fulfilled any of the following 4 criteria within the 3 hour treatment period:

1. 6 episodes of desaturation reported by the caregivers

2. 4 episodes of desaturation for which the infant was stimulated by caregivers

3. 2 episodes of desaturation for which the infant was given supplemental "free-flow" $100 \% \mathrm{O}_{2}$ or positive pressure ventilation by mask by caregivers

4. $\mathrm{SpO}_{2}$ of $<80 \%$ for $>3$ minutes continuously reported by the caregivers

Infants who failed a treatment were restarted on their pre-study treatment by a member of the research team for the rest of that 3-hour period before starting their next assigned treatment.

Nurses recorded episodes of desaturation in enrolled infants in bedside observation charts as per standard practice and informed a member of the study team when an infant reached failure criteria during a treatment. One investigator reviewed these charts following completion of the study and recorded the number of desaturation events reported for each treatment period. The same investigator used a software program (Trendcom, Masimo Corporation, Irvine CA, USA) to download the $\mathrm{SpO}_{2}$ and $\mathrm{HR}$ data from the pulse oximeter into a Microsoft Excel spreadsheet (Microsoft 
Corporation, Redmond WA, USA). Blank data associated with an error message (e.g. no cable, no sensor, sensor off) were not analysed. From this data we determined the following outcomes for each infant during each treatment: the number of episodes and total duration of $\mathrm{SpO}_{2}<80 \%$; the number of episodes and total duration of $\mathrm{SpO}_{2}<85 \%$; the number of episodes and total duration of $\mathrm{SpO}_{2}<90 \%$; the duration $\mathrm{SpO}_{2}>95 \%$; and the episodes and total duration of $\mathrm{HR}<100 \mathrm{bpm}$. Data were analysed using SPSS version 20 (SPSS Inc., Chicago IL, USA). The data were non-normally distributed and as outcomes were based on continuous measurements, we compared the related groups using Friedman tests to compare the three treatment groups, and a Wilcoxon signed rank tests to compare pairs of the treatment groups.

\section{RESULTS}

We enrolled 14 preterm infants, 11 (79\%) of whom were male and 13 (93\%) of whom had received a complete course of antenatal steroids prior to delivery (Table 1).

\section{Table 1: Baseline characteristics}

\begin{tabular}{|l|l|}
\hline Gestational age at delivery (wks) $^{\#}$ & $28(27,29)$ \\
\hline Corrected gestational age at participation in study (wks) & $32(31,33)$ \\
\hline Day of life at participation in study ${ }^{\#}$ & $31(23,40)$ \\
\hline Birth weight (g) & $1195(989,1378)$ \\
\hline Weight on participation in study (g) & $1493(1334,1728)$ \\
\hline Duration of invasive ventilation (days) & $2(2,5)$ \\
\hline $\begin{array}{l}\text { Duration of NCPAP (days) } \\
\text { Duration of nasal cannulae oxygen prior to participation } \\
\text { in the study (days) }\end{array}$ & $22(13,33)$ \\
\hline
\end{tabular}




\begin{tabular}{|l|l|}
\hline Antenatal Steroids & $13 / 14$ \\
\hline Male & $11 / 14$ \\
\hline
\end{tabular}

\# Median (Interquartile Range [IQR])

The median (IQR) gestational age at birth of the infants was $28(27,29)$ weeks and they participated in the study at a median (IQR) postmenstrual age of $33(31,33)$ weeks. Prior to being weaned to nasal cannulae gas, all infants received respiratory support (13 with mechanical ventilation and NCPAP, 1 with NCPAP alone). The median (IQR) duration of nasal cannulae oxygen therapy prior to the study was $4(2,4)$ days. Prior to the study 9 infants were on $100 \%$ oxygen and 5 infants were on blended oxygen at flow rates of between 0.05 $-0.2 \mathrm{~L} / \mathrm{min}$.

Two (14\%) infants reached failure criteria during $\mathrm{O}_{2}$ treatment compared to $7(50 \%)$ infants during both air treatment and sham treatment. The 2 infants who failed the $\mathrm{O}_{2}$ treatment also failed both the air and sham treatments. Both of these infants were on higer flow rates and effective FiO2 before starting the study (SPECIFY WHAT THEY WERE ON IF YOU KNOW).

All 7 infants who failed the air treatment also failed the sham treatment (Table 2).

Table 2: Reasons for treatment failure

\begin{tabular}{|c|c|c|c|}
\hline $\begin{array}{l}\text { Total number of patients reaching } \\
\text { treatment failure criteria }\end{array}$ & 2 & 7 & 7 \\
\hline $\begin{array}{c}\text { SpO2 of }<80 \% \text { for }>3 \text { minutes } \\
\text { continuously }\end{array}$ & 1 & 7 & 7 \\
\hline $\begin{array}{l}2 \text { episodes of desaturation requiring } \\
\text { positive pressure ventilation or } \\
\text { supplemental oxygen }\end{array}$ & 1 & 0 & 0 \\
\hline
\end{tabular}


Infants generally failed early in the treatment period with a median (IQR) time to treatment failure of $47(25,69)$ minutes with oxygen treatment, $16(5,69)$ minutes with air treatment and $5(4,14)$ minutes with sham treatment. The majority $(15 / 16,94 \%)$ of treatment failures were due to $\mathrm{SpO}_{2}$ of $<80 \%$ for $>3$ minutes continuously as reported by the nursing staff; in all cases this was confirmed by the pulse oximetry data. The sole other treatment failure was when an infant had 2 episodes of desaturation treated with supplemental "free-flow" $\mathrm{O}_{2}$. One infant in each treatment group had a single episode of desaturation treated with supplemental "free-flow" $\mathrm{O}_{2}$ for a short period of time and did not reach treatment failure criteria.

There were few missing oximetry data points [mean (SD) of 18 (31) points of 5400 data points recorded in each treatment period, or $0.3(0.6) \%$ of data] and the number did not differ between the three treatments. Among the infants who completed the 3-hour study periods, there were fewer episodes of $\mathrm{SpO}_{2}<80 \%$ during $\mathrm{O}_{2}$ treatment when compared to both air treatment and sham treatment (Table 3).

Table 3: Oxygen saturation $\left(\mathrm{SpO}_{2}\right)$ and heart rate (HR) analysis

\begin{tabular}{|c|c|c|c|c|}
\hline Number of episodes of $\mathrm{SpO}_{2}<90 \%{ }^{\#}$ & $35(15,50)$ & $114(67,172)$ & $148(114,163)$ & $0.004^{*}$ \\
\hline Duration (mins) $\mathrm{SpO}_{2}<90 \%{ }^{\#}$ & $9(3,19)$ & $50(15,79)$ & $52(24,63)$ & $0.005^{*}$ \\
\hline Duration (mins) $\mathrm{SpO}_{2}>95 \%{ }^{\#}$ & $112(78,152)$ & $10(0,83)$ & $7(4,51)$ & $0.005^{*}$ \\
\hline Number of episodes of $\mathrm{HR}<100^{\wedge}$ & $4(4)$ & $2(2)$ & $1(1)$ & 0.368 \\
\hline Duration (mins) $\mathrm{HR}<100^{\wedge}$ & $1(1)$ & $0(0)$ & $0(0)$ & 0.165 \\
\hline Duration (mIns) $\mathrm{SpO}_{2}<85 \% "$ & $2(1,6)$ & $14(2,23)$ & $12(2,21)$ & $0.005^{\uparrow}$ \\
\hline
\end{tabular}

\# Median (IQR) ^Mean (SD) 
* Statistical significance- Friedman test. In all cases of statistical significance Wilcoxon signed rank test showed a statistically significant difference between Oxygen and Sham treatment and Oxygen and Air treatment, with no statistically significant difference between Air and Sham treatment

Similarly there were fewer episodes of desaturation and shorter duration spent with $\mathrm{SpO}_{2}$ $<85 \%$ and $<90 \%$. There were no statistically significant differences between the air and sham treatments in the number of episodes of and duration of desaturation below $80 \%$, $85 \%$ or $90 \%$. Infants had $\mathrm{SpO}_{2}>95 \%$ for longer when treated with $\mathrm{O}_{2}$ compared to air and sham treatment. Episodes of bradycardia, and nurse reported desaturations were few, and did not differ between the treatment periods.

\section{DISCUSSION}

Oxygen is commonly given to preterm infants via nasal cannulae rather than by incubator or headbox as it facilitates parent interaction, nursing care, and feeding. However, it is a largely unstudied treatment, and weaning practices and delivery methods vary widely.

While studies have demonstrated that in preterm infants nasal cannulae delivering gas at flow rates of $1.0-2.5 \mathrm{~L} / \mathrm{min}$ can deliver continuous positive airway pressure,[6,7] the use of gas flow at rates of $<1 \mathrm{~L} / \mathrm{min}$ in an attempt to reduce episodes of apnoea or desaturation has not been studied.

Our study has limitations and only applies to the studied population. The effect of the flow rate may be dependent on its magnitude and we thus do not know if our findings apply at 
rates $>0.1 \mathrm{~L} / \mathrm{min}$. We studied a small number of infants; however, as this was a crossover study, each infant acted as his/her own control, thereby increasing the power of the study. Also, the masked nature of the intervention and the objective outcome assessment by the pulse oximeter reduced the potential for bias and confounders to affect the results. This is highlighted by the low number of nurse reported desaturations, when compared to desaturations as recorded by the pulse oximeter. Most desaturations recorded by the oximeter were brief and resolved spontaneously; it is likely that they were felt not to be clinically significant and were thus not recorded by nursing staff.

It is possible that the sham treatment we used in this study - nasal cannulae disconnected from any flow of gas - could cause a degree of nasal obstruction, and may therefore be worse than no treatment. While we do not use this intervention in clinical practice we felt it necessary to use it in this study to maintain blinding of the caregivers while providing a "washout" period between the air and $\mathrm{O}_{2}$ treatments. We find it interesting that low-flow air was no better than this sham.

Hyperoxia has adverse effects in preterm infants; consequently, we target $\mathrm{SpO}_{2}$ ranges with an upper limit of $95 \%$ when giving supplemental $\mathrm{O}_{2}$ to preterm infants in clinical practice. We did not use this limit in this study as we thought it was important to maintain a constant flow of gas for the duration of the study and considered the concentration of $\mathrm{O}_{2}$ used relatively low (the effective $\mathrm{FiO}_{2}$ generated with $0.1 \mathrm{~L} / \mathrm{min} 100 \% \mathrm{O}_{2}$ has been calculated to be approximately 0.28 in a $1.5 \mathrm{~kg}$ infant, [5]) and the period for which it was administered 
relatively short. While we have demonstrated that low-flow oxygen therapy is beneficial in treating episodes of desaturation, it should be noted that infants had $\mathrm{SpO}_{2}>95 \%$ for a large proportion of the time they were treated with $0.1 \mathrm{~L} / \mathrm{min} 100 \% \mathrm{O}_{2}$ (a median of 112 minutes, compared to 10 and 7 minutes in the air and sham treatment groups respectively). As $\mathrm{SpO}_{2}$ $>95 \%$ would have caused an alarm and prompted a reduction in the amount of supplemental $\mathrm{O} 2$ given in routine practice in our nursery, this illustrates the effect of using supplemental oxygen without an upper target $\mathrm{SpO}_{2}$. Despite the high $\mathrm{SpO}_{2}$ during this treatment, infants had a small number of desaturations with $\mathrm{SpO}_{2} \leq 80 \%$, and more frequent desaturations with $\mathrm{SpO}_{2} \leq 85 \%$ (I DON'T UNDERSTAND THIS - INFANTS STILL HAD SpO $<85$ \& $80 \%$ WHILE RECEIVING $100 \%$ O2?) This highlights the potential difficulties in targeting oxygen saturations in clinical practice. When using $\mathrm{SpO}_{2}$ targets in clinical practice, it is likely that infants will be exposed to more frequent episodes, and a longer duration, of desaturation attempting to avoid the harmful effects of hyperoxia. The adverse effects of brief episodes of desaturation are debated but the recently published studies would suggest prolonged exposure to low $\mathrm{SpO}_{2}$ is harmful. $[8,9]$

Our study demonstrates that the flow of gas is not important in preventing desaturation at $0.1 \mathrm{~L} / \mathrm{min}$ and that the oxygen is the major factor in preventing desaturation. It is reasonable, then, to surmise that weaning $100 \%$ oxygen to a minimal flow rate (e.g. $0.05 \mathrm{~L} / \mathrm{min}$, equivalent to an effective FiO2 of $0.23-0.24$ in a $1.5 \mathrm{~kg}$ baby), rather than maintaining a higher flow rate and weaning the oxygen concentration, may be the more 
appropriate method of weaning infants from low-flow oxygen, provided appropriate target oxygen saturations are used.

\section{CONCLUSIONS}

Our study demonstrates that in preterm neonates receiving supplemental oxygen for low $\mathrm{SpO} 2$, air given at $0.1 \mathrm{~L} / \mathrm{min}$ via nasal cannulae is no better than sham treatment in preventing and limiting the duration of desaturation, while $0.1 \mathrm{~L} / \mathrm{min} 100 \% \mathrm{O} 2$ is superior to both. This demonstrates that at a flow rate of $0.1 \mathrm{~L} / \mathrm{min}$, the flow of gas is not important in the treatment of desaturation in this population of preterm infants. Infants had $\mathrm{SpO}_{2}>95 \%$ for large proportion of the time they were treated with $0.1 \mathrm{~L} / \mathrm{min} 100 \% \mathrm{O} 2$, illustrating the importance of an upper target $\mathrm{SpO}_{2}$ even with low-flow oxygen. 


\section{REFERENCES}

1. Nath P, Ponnusamy V, Willis K, et al. Current practices of high and low flow oxygen therapy and humidification in UK neonatal units. Pediatr Int 2010;52:893-4.

2. Kloor TH Jr, Carbajal D. Infant oxygen administration by modified nasal cannula. Clin Pediatr (Phila) 1984;23:447-449

3. Benaron DA, Benitz WE. Maximizing the stability of oxygen delivered via nasal cannula. Arch Pediatr Adolesc Med 1994;148:294-300

4. Finer NN, Bates R, Tomat P. Low flow oxygen delivery via nasal cannula to neonates. Pediatr Pulmonol 1996;21:48-51.

5. Walsh M, Engle W, Laptook A, et al. Oxygen delivery through nasal cannulae to preterm infants: can practice be improved? Pediatrics 2005;116:857-61.

6. Locke RG, Wolfson MR, Shaffer TH, et al. Inadvertent administration of positive enddistending pressure during nasal cannula flow. Pediatrics 1993;91:135-138.

7. Sreenan C, Lemke RP, Hudson-Mason A, et al. High-flow nasal cannulae in the management of apnea of prematurity: a comparison with conventional nasal continuous positive airway pressure. Pediatrics 2001;107:1081-1083.

8. SUPPORT Study Group of the Eunice Kennedy Shriver NICHD Neonatal Research Network. Target ranges of oxygen saturation in extremely preterm infants. $N$ Engl J Med 2010;362:1959-1969

9. Stenson B, Brocklehurst P, Tarnow-Mordi W; U.K. BOOST II trial; Australian BOOST II trial; New Zealand BOOST II trial. Increased 36-week survival with high 
oxygen saturation target in extremely preterm infants. N Engl J Med

$2011 ; 364: 1680-2$ 